\title{
Vision based Real Time Hand Gesture Recognition Techniques for Human Computer Interaction
}

\author{
Archana S. Ghotkar \\ Pune Institute of Computer Technology \\ University of Pune \\ Pune. (INDIA)
}

\author{
Gajanan K. Kharate, PhD. \\ Matoshri College Of Engineering and Research Centre \\ University of Pune \\ Nashik. (INDIA)
}

\begin{abstract}
Natural Interface with Computer using the intelligent approaches is the need of Human Computer Interaction (HCI)applications. In this paper, three techniques were proposed and experimented for interaction with Desktop/Laptop with static hand gesture. All these techniques were using real time approach with different feature |descriptors such as Fourier Descriptor(FD), 7 Hu moments, |Convex Hull and Finger Detection. Real time Recognition efficiency was calculated with respect to recognition time for FD and $7 \mathrm{Hu}$ |moments. The 300 samples were trained and stored into database for recognition. For unknown user average recognition time was required $1.7 \mathrm{sec}$ using $\mathrm{FD}$ as a feature and $4.6 \mathrm{sec}$ using $7 \mathrm{Hu}$ moments and recognition efficiency was achieved $96 \%$ and $98 \%$ using $7 \mathrm{Hu}$ and FD respectively. In the second technique New Finger detection algorithm was developed and experimented with Hand tracking system(HTS). In this approach, system was working in dynamic background but gives better result in static background. In the third approach, 3-D Kinect camera was used where hand segmentation was achieved using depth image and finger detection were calculated using Convex Hull. In this approach hand segmentation became easier than first two techniques. With all these approaches feature extraction using $7 \mathrm{Hu}$ and FD can be extend for any other HCI application including sign language recognition. Finger counting algorithm can be combined with other descriptor in complex hand sign as a feature. Currently system is |working on static hand gestures, further it will be extended to dynamic hand gesture recognition for Indian sign language interpretation.
\end{abstract}

\section{General Terms:}

Hand Gesture Recognition, Hand segmentation, Hand tracking.

\section{Keywords:}

Human Computer Interface(HCI), Fourier Descriptor(FD), $7 \mathrm{Hu}$ moments, Finger detection, Convex Hull.

\section{INTRODUCTION}

One of the objective of any HCI system is its natural interface. Among other human body part, hand gesture is most natural and powerful communication modality for interaction with computer which needs to be fully explored for HCI. There are two major approaches for hand gesture recognition: Data Glove, Vision based. Each approach is having its limitation and advantages, but vision based approaches are more feasible as compared to data glove as user need not to wear cumbersome device like data glove.[1][2] Many researchers are working on Intelligent application of HCI such as Intelligent Homes/Offices[3],
Intelligent Games [4], Sign language recognition [5] and many more. Thomas and Jaron[6] developed hand-machine interface device that provides real-time gesture, position and orientation information. They used DataGlove containing flex sensors which measure finger blending, positioning and orientation systems and tactile feedback vibrators. William Freeman and Craig Weissman[7] introduced Television control by hand gesture. In their system computer controls the television set through serial port commands. The user uses only one gesture: the open hand, facing the camera and he can control television by moving hand. Interpreting human behavior to understand his cultural background is one of the applications of HCI. Matthias et al. [8] introduced this application with major challenges such as grasping culture as a computational term and inferring the userś cultural background by observable measures. Such an application can be interpreted of the standard language textbook to allow for a deeper understanding of the communication processes that could be achieved by just learning the grammar and words. Afshin et al. [9] proposed algorithms and application for using hand as an Interface device in virtual and physical spaces. They proposed set of applications such as virtual drawing, 3-D model construction and 3-D virtual marble game with hand interface. Christian and Berard[10] described techniques for bare hand interaction with computer and tested on application such as controlling presentation with hand postures, paint virtually onto the wall.

With different applications of HCI, one of the application of Desktop/Laptop interaction with hand interface is chosen for experimentation of algorithms. Natural Interface is preferred to avoid traditional input devices such as keyboard and mouse is the state of the art of HCI application. The same application can be extended for the replacement of mouse by tracking mouse pointer with the tip of the hand finger. Here, five classes of static hand |gestures are used for opening most frequently required windows applications in real time.

In the proposed work three techniques are explored using vision based approach. In the first technique FD and $7 \mathrm{Hu}$ moments were used as a feature descriptor for recognition. In second technique the new Finger Counting algorithm was developed and experimented on HTS [12] system for the same application. Using Convex Hull method and 3D Kinect camera, third technique was explored for the same. The use of Kinect camera made this algorithm easy for pre-processing such as subtraction of complex background and skin color detection.

The organization of the paper is as follows: Section 2 describes the anticipated data-set used for the system. Methodology is explained in section 3 where three different techniques for hand posture recognition are described in detail with experimental results. Conclusion and Future work is given in section 4. 


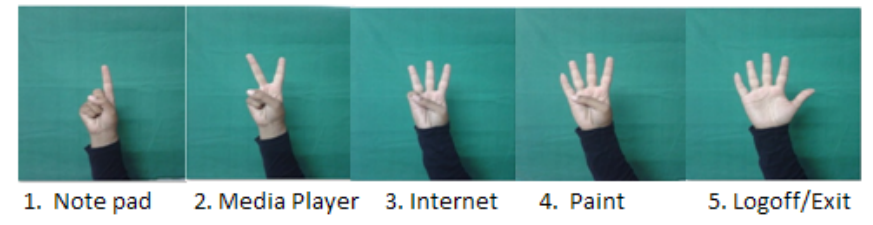

Fig. 1. Anticipated Data-set

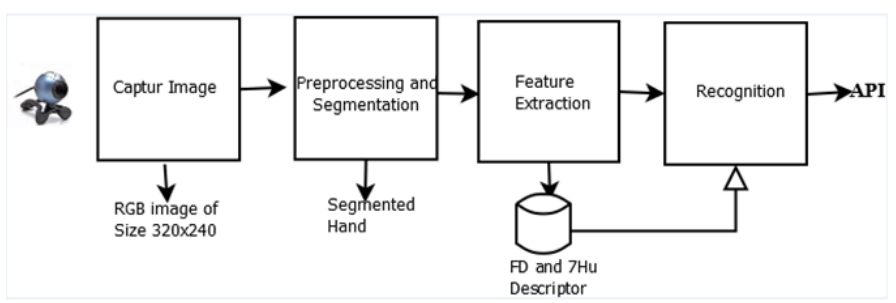

Fig. 2. System Overview

\section{DATA-SET}

Design of data-set of any HCI application is the essential factor as, it should posses naturalness and should not requires extensive |training to user and memorization of hand gestures. Considering these aspect anticipated data set was designed for its user friendliness interactive system. Figure (1) shows anticipated |gesture data set for the proposed system.

\section{METHODOLOGY}

The proposed algorithms were developed for static hand gesture recognition and tested for handling windows applications on Desktop/Laptop. Currently three techniques are proposed and experimented in real time. Vision based hand gesture |recognition is having challenges such as variable lighting condition, dynamic background and skin color detection, considering theses fact algorithms were developed to overcome some of these challenges. Recognition of hand gesture executes windows applications such as opening Note pad, Windows media player, Internet explorer and MS-Paint. All algorithms were working on bare hand where user need not to wear any color gloves or data gloves.

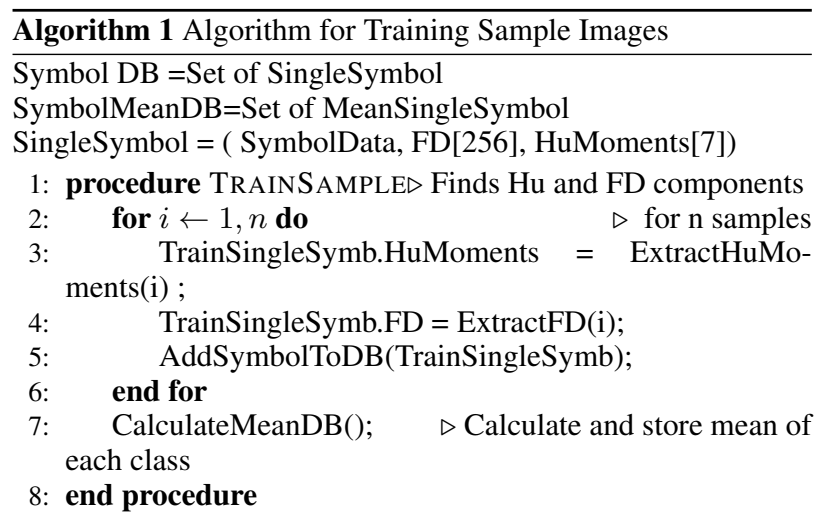

\subsection{Real Time Hand Gesture Recognition Algorithm using $7 \mathrm{Hu}$ moments and Fourier Descriptor}

This algorithm was developed for recognition of hand gesture in real time by keeping static background constraints for faster and accurate recognition. Feature extraction method such as
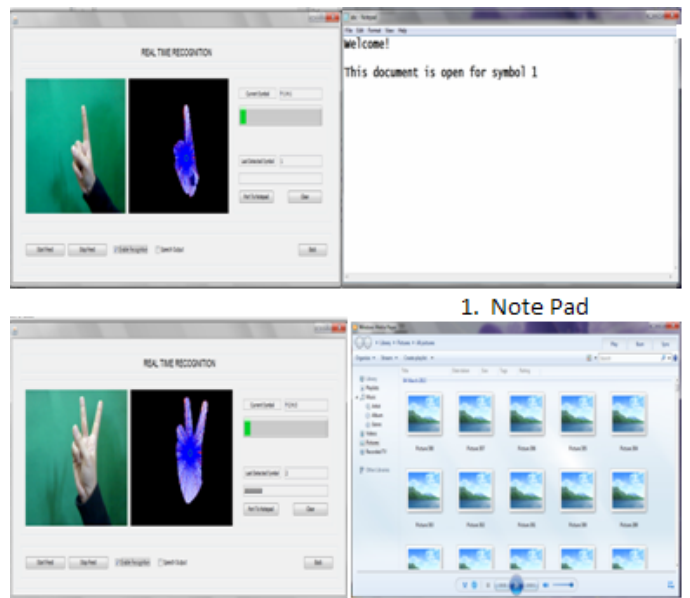

2. Window Media Player
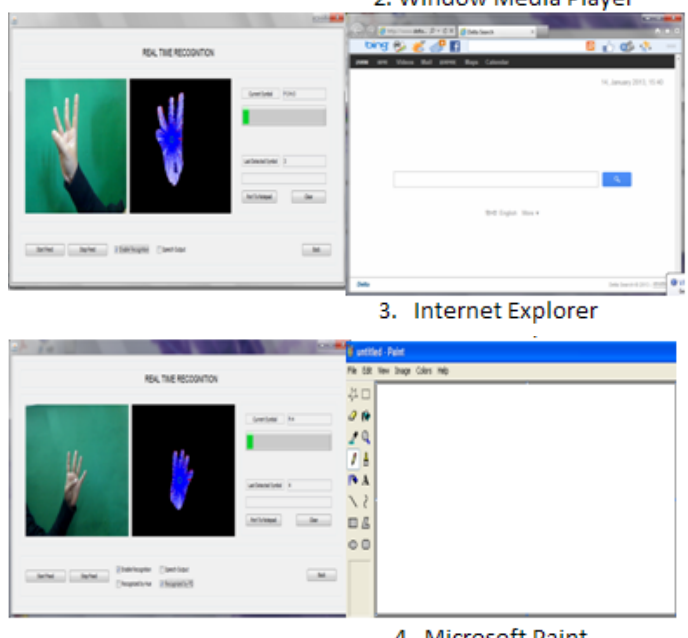

4. Microsoft Paint

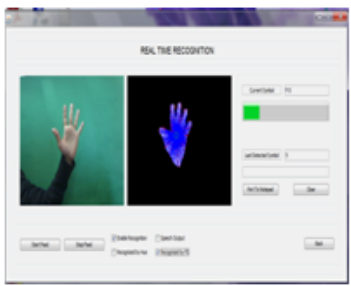

5. Exit

Fig. 3. Hand Gesture Recognition Using 7 Hu moments and FD

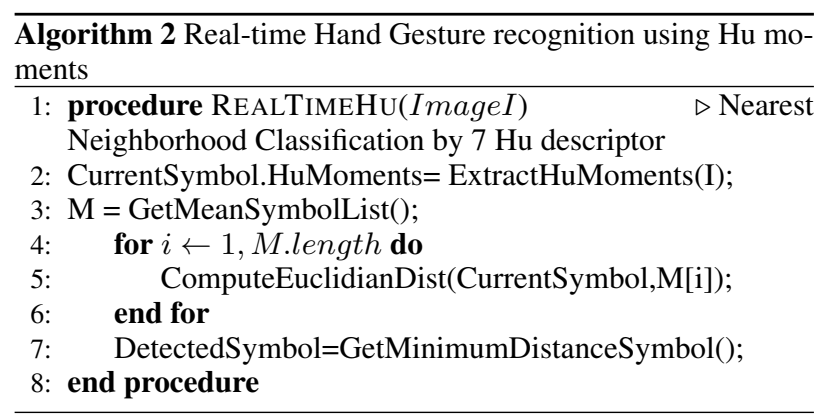




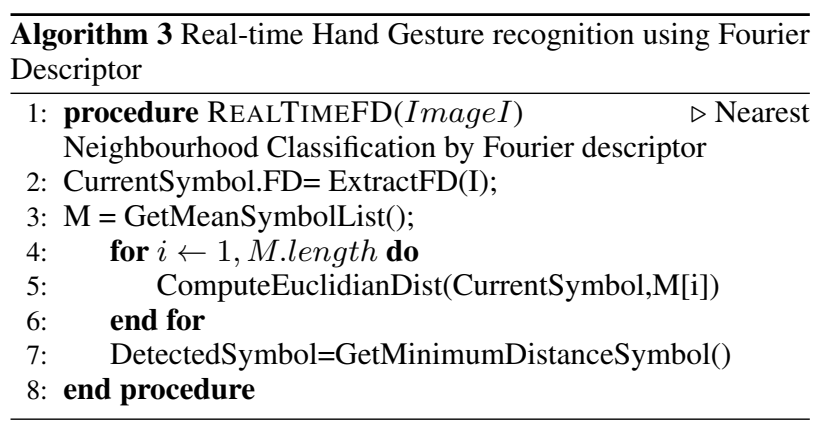

Fourier descriptor and $7 \mathrm{Hu}$ moments were chosen as these methods are invariant to translation, rotation and scale. Algorithm was trained on 300 samples and tested on five unknown users. Performance analysis was done considering recognition time required in real time situation for each class. Nearest neighborhood classification using Euclidean distance was used for recognition purpose. Figure (2) describes the system overview.

3.1.1 Pre-processing and Hand Segmentation. Real time image capturing was done with fixed size of 320x240. In preprocessing Gaussian blur and median filtering was applied for smoothing purpose and HSV [12] color space was used for skin color |segmentation.

3.1.2 Feature Extraction. In the proposed algorithm features of the segmented hand gesture were calculated using Discrete Fourier transform(FD) [13] and $7 \mathrm{Hu}$ moments [14] and stored in to the database for further processing. Algorithm (1) describes Training procedure for sample Images

3.1.3 Recognition. Nearest Neighborhood approach was applied for classification and recognition. Mean value of each class on training sample was calculated and stored for recognition purpose and compared with unknown user. Algorithm (2) and Algorithm (3) describes recognition process with $\mathrm{Hu}$ and FD descriptor. Performance analysis was done for both the descriptors in terms of accuracy and time required for recognition. The Recognition accuracy was calculated by Equation (1) where currently 10 sample test cases are considered for each class in real time. Equation (2) gives average accuracy of HCI system for total number of classes

$$
\begin{gathered}
\operatorname{accuracy}(\%)=\frac{\text { CorrectClassification }}{\text { TotalTests }} * 100 \\
\operatorname{avg}=\sum_{i=1}^{n} \frac{\text { accuracy }}{n}
\end{gathered}
$$

where $\mathrm{n}$ is total number of classes.

3.1.4 Experimental Results. The vision-based hand gesture recognition system also needs to meet the requirements lincluding real-time performances, accuracy and robustness. Here, performance was measured in terms of time (in seconds). For static background with fixed distance $(40 \mathrm{~cm})$ from camera, $7 \mathrm{Hu}$ moments and Fourier descriptor were giving 94\% and $96 \%$ accuracy respectively. If signer distance is constant from camera, classification rate is increased. Figure (3) shows experimental results in real time. Figure (4) and Figure (5) shows performance analysis graph of recognition using $\mathrm{Hu}$ moments and FD respectively. In the graph five test cases are shown on five lunknown users with recognition time required for each class. Average time required for hand gesture recognition using Fourier descriptor was $1.7 \mathrm{sec}$ and using $7 \mathrm{Hu}$ moments was $4.6 \mathrm{sec}$. Figure (6) and Figure (7) shows recognition rate using $7 \mathrm{Hu}$ and FD on 10 test cases for each class in real time.

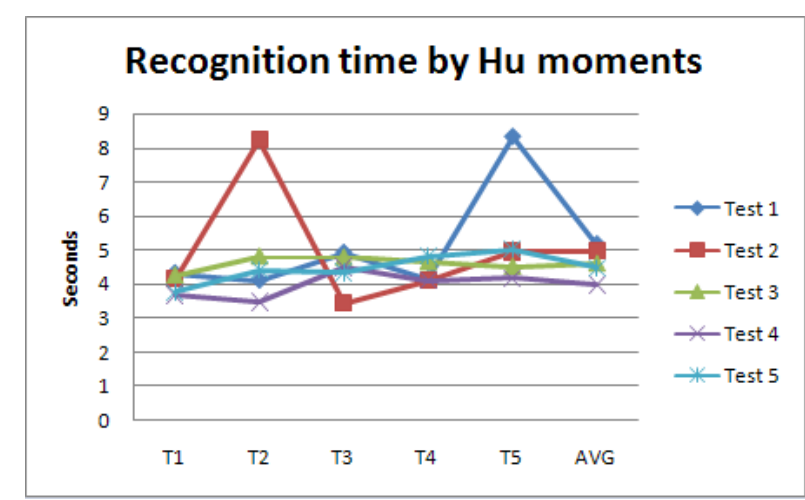

Fig. 4. Performance Analysis Using Hu moments

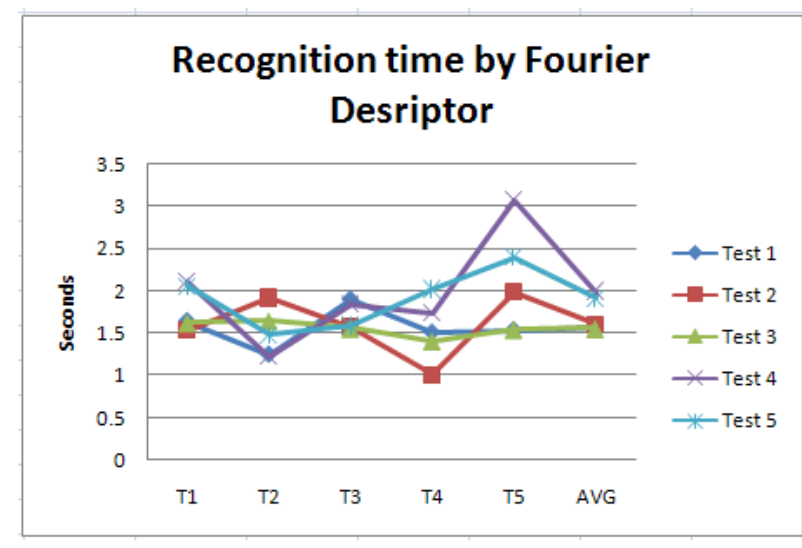

Fig. 5. Performance Analysis Using FD

\subsection{Real time Hand Tracking and Recognition using New Finger Detection Algorithm}

In this technique new algorithm was developed for counting number of finger raised. Here Hand tracking and segmentation(HTS)[12] had been developed under complex background for efficient bare hand segmentation and Finger count algorithm was experimented on segmented hand contour. Algorithm gave the total count of raised finger by analyzing segmented image. In this technique Contour image was scanned from left to right and right to left for detection of finger by analyzing pixel value and finger count was interpreted. Based on finger count value, respective application executed. Algorithm (4), Algorithm (5)and Algorithm (6) describes its working in detail. Figure (8) shows sample results of this algorithm.

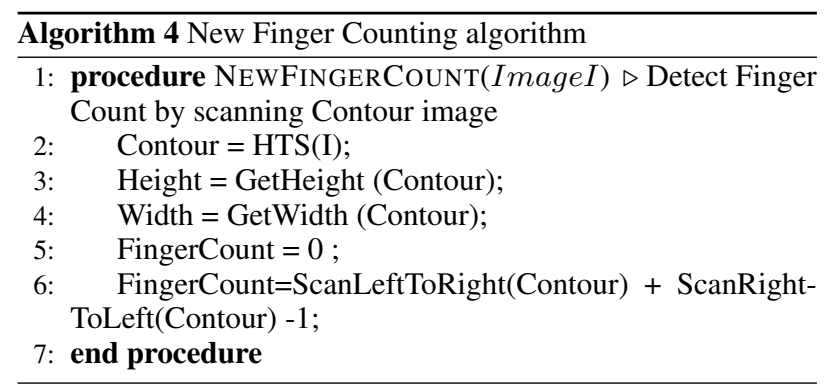



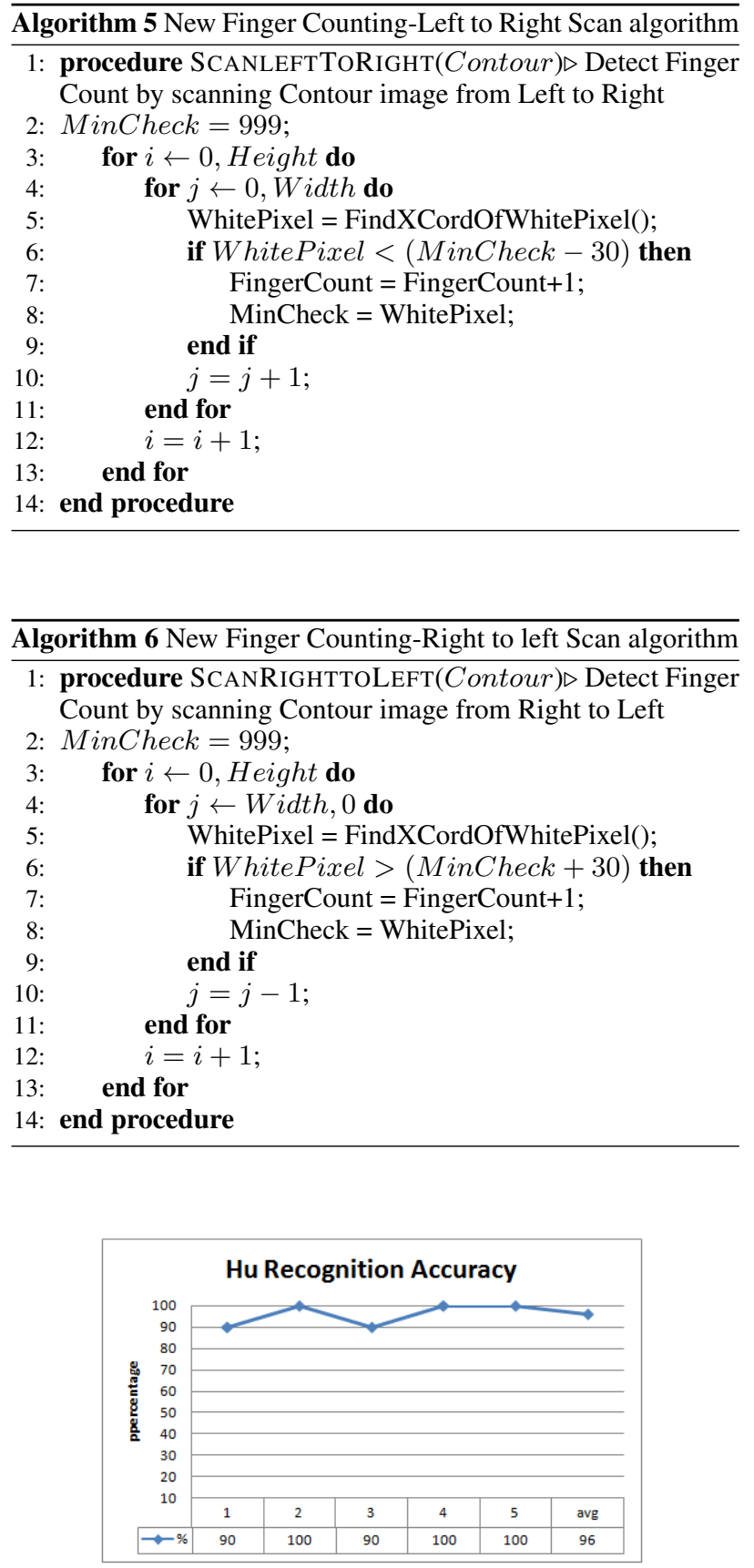

Fig. 6. Recognition accuracy Using 7 Hu moments

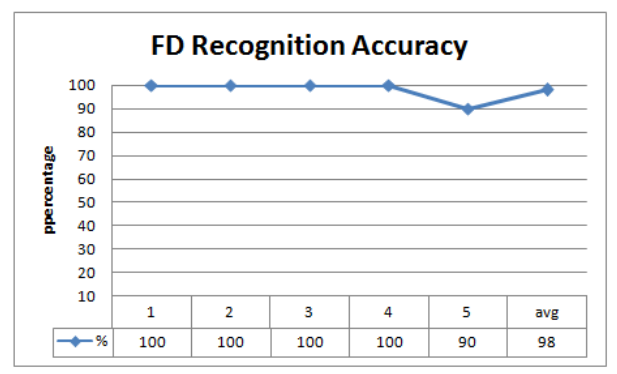

Fig. 7. Recognition accuracy Using FD moments

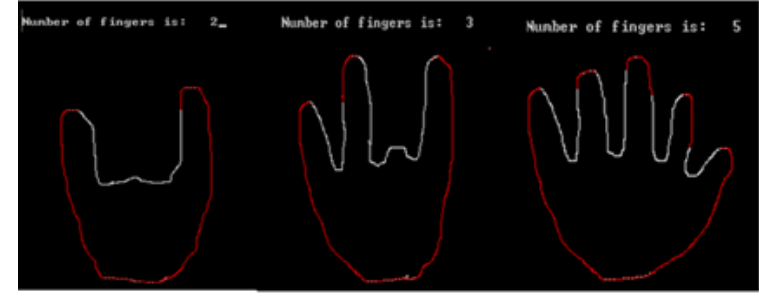

Fig. 8. New Finger Detection algorithm- sample results

\subsection{Real time Hand Gesture Recognition using Kinect camera}

In this approach Microsoft Kinect sensor 3D camera was used for hand recognition. Depth image was used for segmentation. Convex hull OpenCV function was used to detect number of defects(concavities) on hand and stored into the defect array. Based upon number of defects, finger count was determined.

3.3.0.1 Convex Hull. [17] Convex Hull is a region based structural method for shape representation. The prime objective of using Convex Hull on segmented hand is to get convex deficiency of an image. Convex Hull need to be calculated by boundary |tracing or using morphological operation. Polygon approximation was used for extracting Convex Hull to reduce computational time. The extraction of convex hull found significant convex deficiencies along the boundary. The hand shape was represented by a defect |array of concavities. Algorithm (7) describes the working using convex Hull.

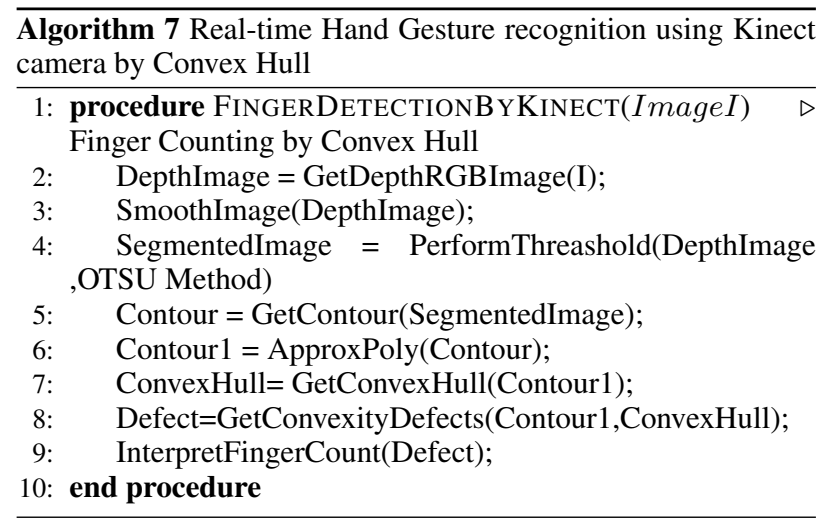

3.3.1 Experimental results. In vision based approach most of the work need to do on pre-processing such as, removing complex background, detecting skin color with simple two dimensional web camera. The main objective of using 3-D Kinect camera was to simplify the task of pre-processing by capturing depth image for hand segmentation.

Figure(9) shows Experimental Results using Convex Hull feature on Kinect camera.

\section{CONCLUSION AND FUTURE WORK}

In this paper three algorithms are developed and tested for HCI application for handling windows most frequently required application with the help of hand gesture. The first two algorithms are tested on 2D web camera of resolution 2Mpixel where as third algorithm is tested on 3D Kinect Camera where depth information is used for segmentation. The same system can be easily extend for replacement of mouse using Kinect camera with the help of skeleton viewer. The average recognition accuracy and time for FD as a feature is better than $7 \mathrm{Hu}$ moments. Finger detection and counting algorithm giving raised finger count, 


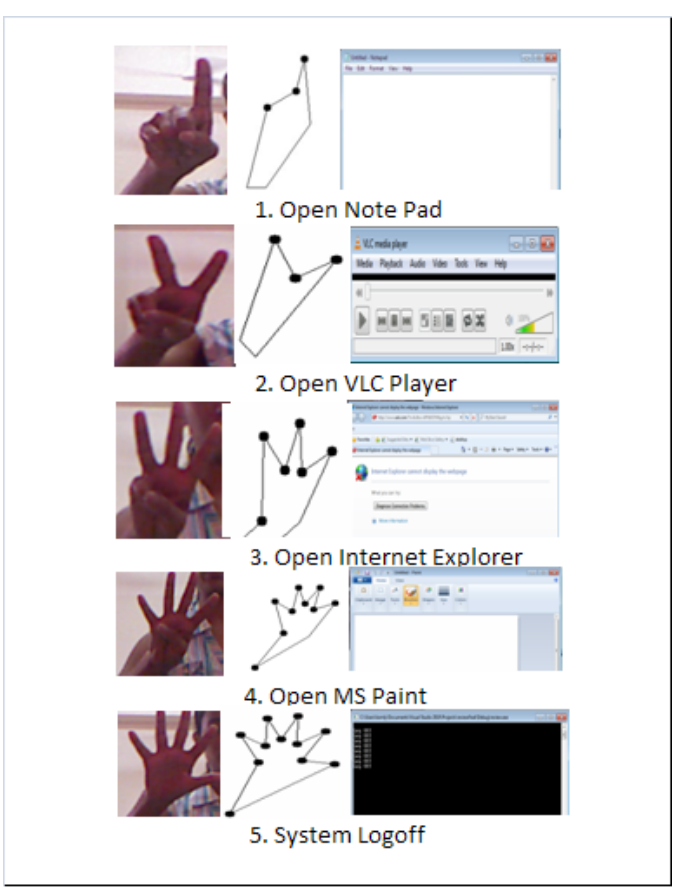

Fig. 9. Hand Gesture Recognition Using Kinect Camera

Table 1. Observations on working of three techniques

\begin{tabular}{|l|l|}
\hline Algorithm & Advantages and Limitations \\
\hline FD,7 Hu features: & $\begin{array}{l}\text { Advantages: i)Invariant to rotation, translation and } \\
\text { scale. ii)Applied to any complex sign. Limitations: } \\
\text { i) Working on static background ii)Training data set } \\
\text { is required . iii) Efficient classifier will required for } \\
\text { large class of data. }\end{array}$ \\
\hline Finger Detection: & $\begin{array}{l}\text { Advantages: i)Training Samples are not required. } \\
\text { ii)Invariant to rotation, translation and scale. } \\
\text { iii) Fast processing due to both side processing. Lim- } \\
\text { itations: i)Only used for finger raised or vertical line } \\
\text { count. }\end{array}$ \\
\hline Convex Hull : & $\begin{array}{l}\text { Advantages:i)Use of 3 D camera simplified task of } \\
\text { segmentation. } \\
\text { ii) Dynamic background subtraction. } \\
\text { iii) Convex Hull method gives accurate defect of hand } \\
\text { shape. iv) Can be used for motion tracking and detec- } \\
\text { tion. Limitations: i)Kinect camera is very sensitive } \\
\text { so creates a problem for robustness. ii)Constant dis- } \\
\text { tance is required to achieve accuracy. }\end{array}$ \\
\hline
\end{tabular}

where training data set is not required. Convex Hull method gives accurate defects of hand in real time, so raised finger can be easily |determined |without training data set. The major objective of this work is to extend the work for sign language interpretation to aid deaf and dumb people. Currently system is working on static hand gesture and different features are generated and tested on different classes of hand gestures. The observation is that all algorithms are robust and accurate under static background and constant |distance of camera and signer.

Table (1) discusses observations of all these techniques with its advantages and limitations. The different generated features such as $7 \mathrm{Hu}$ moments, FD and count of raised finger could definitely used for complex hand sign of sign language. In this paper nearest neighborhood classification using Euclidean distance is developed for recognition but in future efficient classification and recognition algorithm such as Navies Bayesian classification[18][19], support vector machine[20][21] [22] or Genetic algorithm[23] can be used for large class of data.

\section{ACKNOWLEDGMENT}

The authors are thankful to the Department of Science and Technology, Ministry of Science and Technology, New Delhi for supporting this non-commercial research under the Fast Track Scheme for Young Scientists (SR/FTP/ETA-18/08).

\section{REFERENCES}

[1] Noor Saliza,Jamilin Jais, "Hand Gesture Recognition using Hidden Markov Models:A Review on Techniques and Approaches",

[2] Pragati Garg,Naveen Aggarwal and Sanjeev Sofat, "Vision based Hand GestureRecognition", PWASET,Vol.37,pp.1024-1029,2009.

[3] S.Meyer and A.Rakotonirainy, "A survey of research on context-aware homes", Australasian Information Security Workshop Conference on ACSW Frontiers, pp. 159-168, 2003.

[4] K.Salen and E. Zimmerman, "Rules of Play : Game Design Fundamentals", MIT press, Cambridge, 2003.

[5] Britta Bauer, Karl-Friedrich, "Towards an Automatic Sign Language Recognition System Using Subunits", GW 2001,LNAI 2298, Springer-Verlag Berlin Heidelberg pp3447,2002.

[6] Thomas G.Zimmerman, Jaron Lanier, "A Hand Gesture Interface Device”, ACM-089791-213-6, pp. 189-192, 1987.

[7] William T. Freeman and Craig D. Weissman, "Television control by hand gestures",

[8] Matthias Rehm, Nikolaus Bee, Elisabeth Andre "Wave Like an Egyptian- Accelerometer Based Gesture Recognition for Culture Specific Interactions", British Computer Society,2007.

[9] Afshin Sepehri, Yaser Yacoob, Larry S. Davis "Employing the hand as n Interface Device", Journal of Multimedia, Vol.1 No. 7, ,Academy Publisher,pp.18-29,2006.

[10] Christian von Hardenberg, Francois Berard, "Bare-Hand Human Computer Interaction", Proceedings of the ACM Workshop on Perceptive User Interfaces, Orlando, Florida, USA, 2001.

[11] Elena Sanchez-Nielsen, Luis Anton-Canalis and Mario Hernandz-Tejera, "Hand Gesture Recognition for Human Machine Interaction", Journal of WSCG, Vol.12, No.1-3, ISSN 1213-6972, 2003.

[12] Archana S. Ghotkar, Gajanan K. Kharate, "Hand Segmentation Techniques to Hand Gesture Recognition for Natural Human Computer Interaction”, International Journal of Human Computer Interaction(IJHCI), Computer Science Journal,Malaysia, Volume (3) : issue(1), pp.15-25.ISSN 2180 -1347,April 2012.

[13] Gang Zhang, Z.M..Ma, Ying He, Tienan Zhao, ”Shape Feature Extraction Using Fourier Descriptors with Brightness in Content-based Medical Image Retrieval ", International Conference on Intelligent Information Hiding and Multimedia Signal Pro- cessing, IEEE Computer Society, 2008.

[14] Yang Quan,Peng Jinye,Li Yulong "Chinese Sign Language Recognition Based on Gray Level Co-Occurrence Matrix and Other Multifeatures Fusion”, IEEE-ICIEA,pp.1569$1572,2009$.

[15] Dengsheng Zhang,Cuojun Lu "Review of shape representation and description techniques", The Journal of The Pattern Recognition Society,Elsevier,pp1-19,2004.

[16] Yun Liu,Zhijie Gan,Yu Sun "Static Hand Gesture Recognition and Its Application based on Support Vector Machines", ACIS International Conference,IEEE Computer Society,pp.517-521,2008. 
[17] Anil K.Jain, Robert P.W.Duin,Mao, ”Statistical Pattern Recognition: A Review”, pp1-105,1999.

[18] Guo-Dong, Anil K. Jain,Wei-Ying Ma, Hong-Jiang Zhang, "Learning similarity Measure for Natural Image Retrieval with Relevance Feedback", IEEE Transactions on Neural Networks, Vol.13, No. 4, July 2002.pp. 811-820.

[19] Shu-fai Wong, Roberto Cipolla, "Continuous gesture recognition using a sparse Bayesian classifier", International conference on pattern recognition, 2006.

[20] Luis Gonzalez, Cecilio Angulo, Franscisco, Juan Antonio Ortega, "A Probabilistic Tri-class Support Vector Machine",
[21] Liu Yun, Zhang Peng "An Automatic Hand Gesture Recognition System based on viola-Jones Method and SVMS", International workshop on Computer Science and Engineering, IEEE Computer Society, 2009,pp. 72-76.

[22] David Mace, Wei Gao, Ayse Coskun, "Improving Accuracy and Practicality of Accelerometer Based Hand Gesture Recognition”, IUI,2013.

[23] Min Pei, Erik Goodman, William Punch, Ying Ding, "Genetic Algorithms For Classification and Feature Extraction? Classification Society of North America”, Classification Society of North America,1995. 